\title{
GENOMICS
}

\section{Diploid genome in 3D}

Dip-C, a descendent of the 3 C method, reveals 3D genome structures of single diploid human cells.

O ver the past decade, researchers have learned that not only the sequence of genome but also the threedimensional (3D) organization of genome is critical to gene regulation and cell function. The advent of chromosome conformation capture (3C) and derivative methods (such as $\mathrm{Hi}-\mathrm{C}$ ) has rapidly expanded knowledge on the $3 \mathrm{D}$ folding of chromosomes and helped scientists decipher the association of 3D structures with gene regulation.

Beyond bulk samples, recent advances in single-cell Hi-C methods, in combination with statistical analysis and structural modeling, have allowed the reconstruction of chromosomes that vary between cells and pinpointed heterogeneity in cell populations. However, single-cell genome reconstruction with high resolution has been limited to haploid cells because of the ambiguities that arise from the two parental chromosomes in diploid genomes. Sunney Xie and his team from Harvard University recently developed a 3C-derived method along with an imputation algorithm, together termed Dip-C, to reconstruct the spatial organizations of diploid genomes at the single-cell level. Dip-C allows the examination of single diploid cells, and has revealed that different cell types exhibit distinct 3D genome structures.

Dip-C, similar to other $3 \mathrm{C}$ methods, relies on proximity ligation of chromatin fragments, but it also incorporates transposon-based amplification of ligated fragments to improve whole-genome coverage and avoid biotin pulldown steps. Compared with existing methods, Dip-C is capable of detecting more chromatin contacts and distinguishing the two parental alleles on the basis of their single-nucleotide polymorphisms.
The authors reconstructed single-cell diploid genomes from a lymphoblastoid cell line derived from a female subject and from primary blood cells from a male subject at $20-\mathrm{kb}$ resolution. With improved spatial resolution, Dip-C can probe the structural differences between the maternal and paternal alleles of imprinted loci, as well as pinpoint the location of genomic variation in the nucleus.

Lei Tang

Published online: 30 October 2018 https://doi.org/10.1038/s41592-018-0208-7

Research papers

Tan, L. et al. Three-dimensional genome structures of single diploid human cells. Science $\mathbf{3 6 1}$, 924-928 (2018).

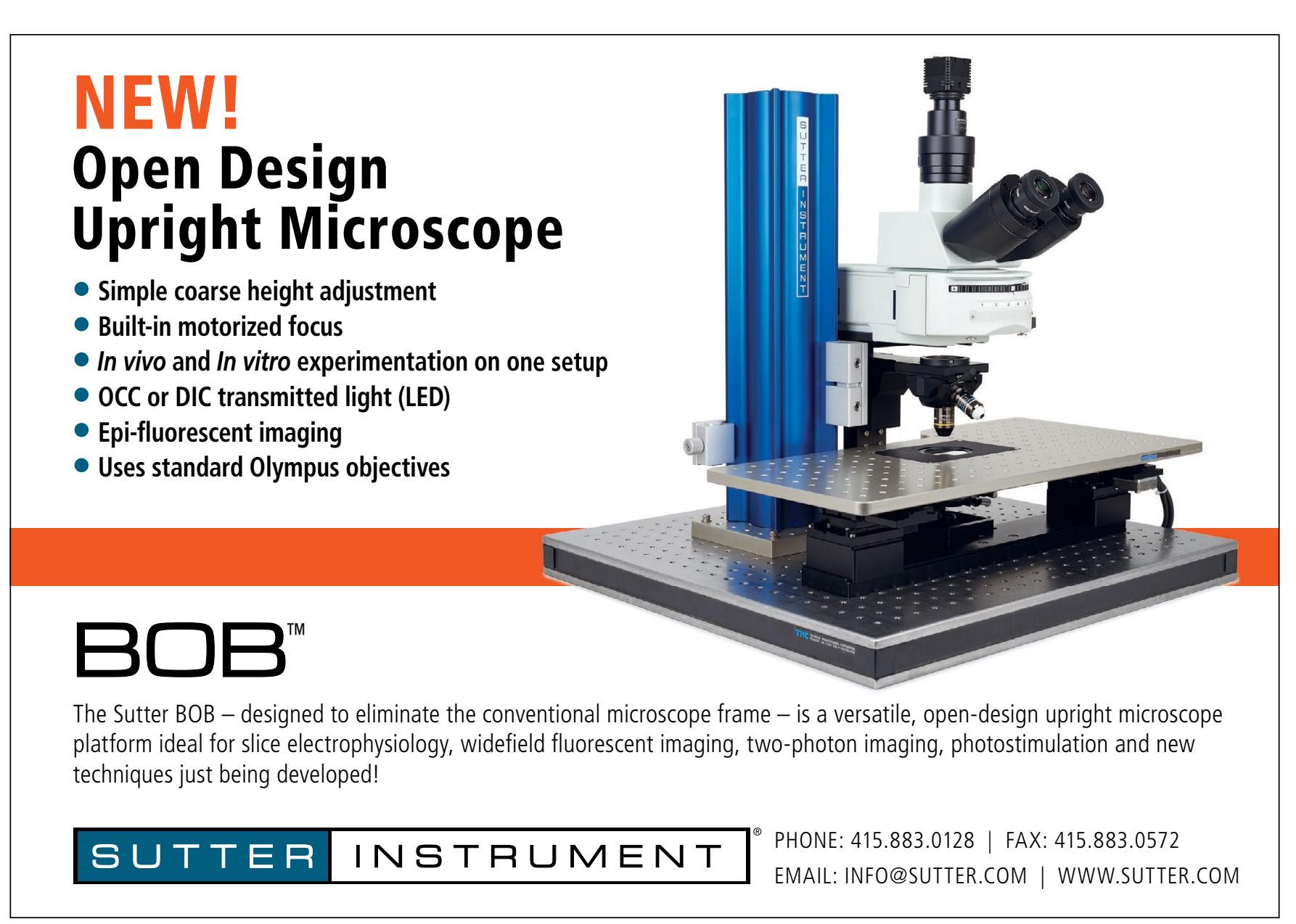

